\title{
(8) \\ Método activo en el desarrollo de competencias matemáticas en niños de la cultura Awajún, Perú
}

\author{
Active method in the development of mathematical competences in Awajún \\ children, Perú
}
Método ativo no desenvolvimento de habilidades matemáticas em crianças da cultura Awajún, Peru

\section{Abdías Chávez-Epiquén ${ }^{1}$ Ketty Marilú Moscoso-Paucarchuco² ${ }^{\bullet}$ Juan Raúl Cadillo-León $^{3}$}

Received: Jan/06/2020 • Accepted: Mar/21/2020 • Published: Jan/31/2021

\section{Resumen}

El objetivo de la investigación fue establecer la relación entre el uso de aprendizaje activo y el desarrollo de competencias del área de matemática en los estudiantes del tercer grado de primaria de la cultura awajún. El diseño de investigación fue preexperimental pretest/postest, enfoque mixto, aplicado y explicativo. La muestra estuvo compuesta por 22 estudiantes de tercer grado de primaria de la cultura awajún, distrito de El Cenepa, provincia Condorcanqui, departamento del Amazonas Perú, 2018. Las técnicas aplicadas fueron observación y prueba pedagógica. Los instrumentos corresponden a la prueba pedagógica y la ficha de observación. Los resultados del pretest indican que no hubo estudiantes que alcanzaran los niveles de logro y logro destacado en las capacidades matemáticas evaluadas. Los resultados postest indican que el $72 \%$ de los estudiantes alcanzaron los niveles de logro y logro destacado posterior a la aplicación de la didáctica basada en el aprendizaje activo. Se concluye que el uso del aprendizaje activo influyó el desarrollo de competencias del área de matemática en los alumnos del tercer grado de primaria de la cultura awajún del distrito de El Cenepa, provincia Condorcanqui, departamento del Amazonas Perú, en 2018.

Palabras clave: Aprendizaje activo; awajún; didáctica; matemática; educación matemática; competencias matemáticas; educación primaria; Amazonas; capacidades matemáticas.

\section{Abstract}

The objective of this paper was to establish the relationship between using active learning and developing mathematical competencies in third grade Awajún students. The research design was mixed, applied and explanatory, pre-test/post-test. The sample was comprised of 22 third grade students from the Awajún culture in the El Cenepa district, province of Condorcanqui, department of Amazonas, Peru, in 2018. Techniques applied were observation and pedagogical tests, while instruments included a pedagogical test

\footnotetext{
Abdías Chávez-Epiquén, $\triangle$ achavez@unca.edu.pe, ㄴ https://orcid.org/0000-0001-5589-5217

Ketty Marilú Moscoso-Paucarchuco, $\$ mpketty1982@gmail.com, (1) https://orcid.org/0000-0003-2097-8658

Juan Raúl Cadillo-León, $\square$ juan.cadilloleon@gmail.com, (ㄱ https://orcid.org/0000-0002-3259-3934

1 Departamento de Administración del turismo y hotelería, Universidad Nacional Ciro Alegría, La libertad, Perú.

2 Escuela de Administración de turismo sostenible, Universidad Nacional Autónoma de Huanta, Huanta, Perú.

3 Escuela profesional de Ingeniería de Sistemas, Universidad Católica Los Ángeles de Chimbote, Chimbote, Perú.
} 
and an observation sheet. According to pretest results, no students reached the meet and master levels in the mathematical skills assessed. The post-test results indicated that $72 \%$ of the students reached the meet and master levels after the application of didactics based on active learning. It is concluded that the use of active learning influences the development of mathematics competencies in Awajún third grade students from the El Cenepa district, province of Condorcanqui, department of Amazonas, Peru, in 2018.

Keywords: Active learning; Awajún; didactics; mathematics; mathematics education; mathematical skills; elementary education; Amazonas; Mathematics competencies.

\section{Resumo}

0 objetivo da pesquisa foi estabelecer a relação entre o uso da aprendizagem ativa e o desenvolvimento de habilidades na área de matemática nos estudantes do terceiro ano do ensino fundamental da cultura Awajún. 0 desenho da pesquisa foi pré-teste/pós-teste pré-experimental, de enfoque misto, aplicado e explicativo. A amostra foi composta de 22 estudantes do terceiro ano do ensino fundamental da cultura Awajún, distrito de El Cenepa, província de Condorcanqui, departamento de Amazonas, Peru, 2018. As técnicas aplicadas foram de observação e de teste pedagógico. Os instrumentos correspondem à prova pedagógica e à ficha de observação. Os resultados do pré-teste indicam que não houve estudantes que atingiram os níveis de desempenho e desempenho notável nas habilidades matemáticas avaliadas. Os resultados do pós-teste indicam que $72 \%$ dos estudantes atingiram os níveis de desempenho e desempenho notável após a aplicação da didática com base na aprendizagem ativa. Conclui-se que 0 uso da aprendizagem ativa influenciou o desenvolvimento de habilidades na área de matemática nos alunos do terceiro ano do ensino fundamental da cultura Awajún do distrito de El Cenepa, província de Condorcanqui, departamento de Amazonas, Peru, em 2018.

Palavras-chave: aprendizagem ativa; Awajún; didática; matemática; educação em matemática; habilidades matemáticas; educação elementar; Amazônia; competências matemáticas.

\section{INTRODUCCIÓN}

El "Laboratorio Latinoamericano de Evaluación de la Calidad de la Educación [LLECE]" en coordinación con la "Oficina Regional de Educación de la UNESCO para América Latina y el Caribe" adelantaron el "Segundo Estudio Regional Comparativo y Explicativo SERCE 2004-2008" (UNESCO \& Laboratorio Latinoamericano de Evaluación de la Calidad de la Educación [LLE$\mathrm{CE}$, 2008). El objetivo del SERCE fue revisar, evaluar y analizar los procedimientos, contenidos y aprendizaje de la enseñanza de la matemática, lectura y ciencias en los niños de los países participantes, quienes se encontraban cursando tercero y sexto grado de Educación Primaria en 2005 y 2006. Aunado a ello, se persiguió identificar los factores, concomitantes o no, que inciden en la obtención de los resultados alcanzados. El estudio fue motivado como consecuencia del diagnóstico UNESCO \& LLECE (2008) referido a los sistemas educativos de la región, donde se expresa los altos índices de deficiencias en el rendimiento escolar. El resultado del Perú en el SERCE ubicó a los estudiantes nacionales con promedios inferiores a los obtenidos por sus homólogos regionales. El análisis indicó que el $15.4 \%$ de los escolares peruanos se encuentran por debajo del Nivel 1 y el $42.42 \%$ se 
encuentra en el Nivel 1. En consecuencia, se recomendó la intervención institucional con el objetivo de corregir los bajos niveles de aprendizaje (UNESCO \& LLECE, 2008). Los resultados del TERCE indicaron que el $15.24 \%$ de los escolares peruanos del tercer grado de primaria se ubicaban debajo del nivel I y el $45.42 \%$ en el nivel I (UNESCO \& LLECE, 2015). Los indicadores expresan los bajos niveles de aprendizaje de los estudiantes en las áreas evaluadas.

Los indicadores de la Evaluación Internacional PISA 2012 (PISA 2014), ubicaron a los países latinoamericanos en los niveles de aprendizaje más bajos en las áreas de matemáticas, comprensión lectora y ciencia. En 2012, PISA situó al Perú en el lugar 65 de los países evaluados, con un puntaje de 368 puntos en el área de la matemática (OCDE, 2014). Posteriormente la evaluación PISA, en 2015, ubicó al Perú en el lugar 62 de 70 países, con un puntaje promedio de 387 , lo que dista de manera importante de los puntajes de los países OCDE (OCDE, 2016). En el año 2015, en el área de matemáticas, donde participaron 72 países el Perú ocupó el puesto 61, por encima del obtenido por los alumnos de Brasil e Indonesia (OCDE, 2016). En el mismo orden, con el objetivo de diagnosticar e intervenir en la mejora del proceso enseñanza-aprendizaje el Ministerio de Educación del Perú aplicó hasta el año 2018 la Evaluación Censal a Estudiantes (ECE). El ECE era convocado durante el último trimestre del año, coincidiendo con el último trimestre del año escolar, bajo la teleología de obtener un índice actualizado sobre el nivel de aprendizaje obtenido por los estudiantes de $2^{\circ}$ y $4^{\circ}$ de primaria y $2^{\circ}$ de secundaria. Los resultados del ECE 2018 arrojaron pocas variantes con las evaluaciones ECE 2016. En el año 2017 no hubo convocatoria para la evaluación ECE.
Según la "Unidad de Medición de la Calidad (UMC)" del Ministerio de Educación, los resultados de las evaluaciones censales en los tres últimos años han reportados leves mejorías en cuanto al desempeño de los niños. En el año 2015, el $26.6 \%$ de los escolares del segundo grado se encontraban en un nivel satisfactorio, el $42.3 \%$ en el de proceso y el $31.0 \%$ en el inicial (Ministerio de Educación del Perú [Minedu], 2015a). En el año 2016, el $34.1 \%$ de los niños de segundo grado de primaria se encontraban en un nivel satisfactorio de desempeño, mientras que $37.3 \%$ en el de proceso y el $28.6 \%$ en el de inicio (Minedu, 2016a). Los resultados nacionales del 2018 para el área de matemáticas en el $2^{\circ}$ grado indicaron que el $14.7 \%$ de los estudiantes se ubicaron en el nivel satisfactorio, 30.3 en el nivel proceso y $55 \%$ en el de inicio (Minedu, 2018).

Los resultados del ECE a lo largo de los años evidencian asimetrías departamentales, provinciales y distritales en los niveles de aprendizaje matemático. Para el año 2016 se registraron distritos donde más del $80 \%$ de los estudiantes se ubicaron en el nivel inicio:100 \% San Antonio de Cachi departamento de Apurímac, 80 \% Kelluyo departamento de Puno, 80 \% Yaquerana 84.1 \% Mazan y $84.4 \%$ Napo en el departamento de Loreto, 88.2 \% Pucacolpa departamento de Ayacucho, $86.8 \%$ Tahuania departamento de Ucayali, $82.9 \%$ Icahuasi departamento Lambayeque. Mientras que hubo distritos donde el porcentaje de estudiantes quienes alcanzaron el nivel de satisfactorio superó el $70 \%$ como los casos de: $100 \%$ Luis Carranza departamento de Ayacucho, $100 \%$ San Gregorio departamento de Cajamarca, $100 \%$ Coayllo, Huancaya, Huangascar y Huantan en la provincia de Lima, $100 \%$ Cochamal, $92 \%$ Cheto y $79 \%$ Luya departamento de Amazonas, $76.9 \%$ Vilcabamba y 
$75 \%$ Virundo departamento de Apurimac, $72.1 \%$ Ticapampa departamento de Ancash, $78.3 \%$ Cabanaconde departamento de Arequipa (Minedu, 2016a). La polarización de resultados es consecuencia de múltiples factores, no obstante, al observar el retroceso nacional en los resultados ECE entre 2016 y la evaluación maestral 2018, se infiere la persistencia de brechas en la enseñanza de la matemática. En el 2018, fue implementada la nueva modalidad de evaluación muestral (EM) para estudiantes de segundo grado de educación primaria, lo que arrojó que 14.7 $\%$ de los estudiantes habían alcanzado las competencias básicas de matemáticas, 30.3 $\%$ se encontraban en proceso y $55 \%$ en el nivel inicio (Minedu, 2018).

En el nivel de la Región Amazonas, en el año 2015 los resultados ECE para el área de matemáticas en estudiantes de segundo grado indican que el $43 \%$ se ubicaban en el nivel satisfactorio, el $49.4 \%$ en el de proceso y el $7.6 \%$ en el nivel de inicio. En el año 2016, el $40.4 \%$ de los niños del segundo grado se encuentran en un nivel satisfactorio, el $52.1 \%$ en el de proceso y el $7.5 \%$ en el de inicio (Minedu, 2016a). El Distrito de El Cenepa, se encuentra ubicado en la Provincia de Condorcanqui de la Región Amazonas. Los resultados de las evaluaciones reportaron que en año 2016, solo el $8.0 \%$ de los niños de segundo grado de primaria se encuentran en el nivel satisfactorio, el $23.9 \%$ en nivel de proceso y el $68.1 \%$ en el de inicio. En el año 2016 , el $10.6 \%$ se encontraba en el nivel satisfactorio en cuanto a su desempeño, el 35.3 $\%$ en el de proceso y el $54.1 \%$ en el de inicio (Minedu, 2016b).

Estos resultados reflejan que un alto porcentaje de niños, quienes aprobaron el segundo grado de primaria, aún no habían logrado las competencias necesarias para desempeñarse adecuadamente en el ciclo correspondiente al tercer grado. En ese orden, diversos autores refieren la necesidad de romper con los esquemas tradicionales de la enseñanza de la matemática (Godino, 2017; Godino et al., 2017; Mota, Henríquez y Oliveira, 2016; Salas, 2018), con el objetivo de promover en el estudiante la empatía por la asignatura fundamentada en la percepción de logro (Zumaeta, Fuster y Ocaña, 2018).

\section{Cultura awajún de El Cenepa}

La etnia awajún pertenece a la familia etnolingüística jíbaro, ubicada en la selva tropical y recorrida por el río Marañon y los denominados "Cinco Ríos" entre ellos el río Cenepa que le dio el nombre al distrito El Cenepa donde se encuentra la localidad Kusu Chico (Burneo, 2018). La población awajún de Kusu Chico fue reconocida como comunidad nativa el 12 de junio del año 1975, ocupa una extensión de $992 \mathrm{Ha}$ (Ministerio de la Cultura, 2018). Las actividades económicas preponderantes son la agricultura, caza y pesca de subsistencia, aunado a la minería artesanal.

La realidad de los niños de la Amazonia posee singularidades en comparación al resto de la población estudiantil del país. Los estudiantes del nivel primaria pertenecientes a la cultura awajún, son hijos de padres de etnia originaria. El desarrollo de estos pobladores confronta situaciones críticas debido a la deforestación y permanente riesgo de la vida y cultura por el despliegue de las actividades petrolera y minera formal e informal. La expansión de la minería informal y las prácticas extractivas basadas en el uso de mercurio y otros minerales pesados amenaza los medios de subsistencia local, como resultado de los altos niveles de contaminación (Osores, Rojas y Manrique, 2012). La prioridad para los padres es la sobrevivencia lo que relega la educación a un 
segundo plano, unido a las condiciones extremas de subsistencia, algunos estudiantes deben caminar entre 3 y 4 días para poder ir a la escuela. La pobreza en esta zona es de $58 \%$ y la extrema pobreza alcanza $29 \%$. La tasa de analfabetismo bordea el $12 \%$ en mayores de 15 años, en las mujeres alcanza hasta el $18 \%$ (INEI, 2018).

\section{Aprendizaje activo de la matemática}

Los estilos de aprendizaje están asociados a las diversas formas en que las personas aprenden. El aprendizaje se distingue por la estrategia que cada persona selecciona preponderando un método sobre otro (Borracci, et al, 2015). Estos estilos se expresan en la preeminencia de mecanismos visuales, lectoescritura, cinestésicos, auditivos o multimodales que utilizan las personas para adquirir, transmitir y utilizar los conocimientos.

El aprendizaje activo comprende la metodología basada en la promoción de la participación del estudiante como prosumidor de conocimiento, por parte del docente (Labrador y Andreu, 2015). Ello comprende la incorporación activa del estudiante en el desarrollo de las actividades inherentes al proceso enseñanza-aprendizaje, que redunden en el desarrollo de habilidades y destrezas en los ámbitos comunicacionales, cooperativos, colaborativos, autónomos, innovación, proyectos y solución de problemas. En ese contexto el estudiante desempeña roles activos en el proceso de aprendizaje, lee, cuestiona, escribe, discute, aplica conceptos, utiliza reglas y principios, resuelve problemas. El aprendizaje activo demanda del docente la planificación de actividades de estimulación continua para que el estudiante individual o colectivamente desempeñe procedimientos de orden superior: análisis, síntesis, interpretación, inferencia, evaluación (Universidad ICESI,
2017). Estos procedimientos son fundamentales para el aprendizaje matemático.

En el ámbito de la Educación Intercultural Bilingüe, el desarrollo de competencias etnomatemáticas persigue que los estudiantes de los pueblos nativos logren desenvolverse con eficacia y eficiencia durante el desarrollo de la vida. (Minedu, 2015b). En ese contexto, el aprendizaje por competencias proyecta el desarrollo de procesos pedagógicos orientados a satisfacer la exigencia social de la reflexividad ciudadana (Solar y Deulofeu, 2017). El ciudadano reflexivo es capaz de construir argumentos que expliquen situaciones y contextos, al recurrir a representación simbólica que exprese perspectivas y posibiliten la solución de problemas fundamentado en herramientas matemáticas. Desde esa perspectiva, el programa curricular de educación primaria del Perú (2016) establece al enfoque centrado en la resolución de problemas como marco teórico-metodológico del proceso enseñanza-aprendizaje orientado hacia el desarrollo de las competencias inherentes a los dominios: Número y operaciones, cambio y relaciones, geometría y estadística y probabilidad (Minedu, 2015b). En ese orden, el currículo se inscribe dentro de la perspectiva del aprendizaje activo, donde el docente funge de promotor en el ejercicio de la abstracción por el estudiante. Las experiencias del estudiante posibilitan la construcción de conocimiento mediante la interacción e intercambio de información con los pares, el docente o la reflexión individual sobre temáticas concretas. Ello induce hacia la proposición de soluciones y estrategias alternas ante situaciones dadas o experimentadas, fundamentadas en aplicaciones matemáticas (Minedu, 2016a).

En las escuelas interculturales bilingües (EIB), y específicamente en la I. E. 
N. ${ }^{\circ} 16348-B$ de la localidad de Kusu Chico del Amazonas, prevalece la educación fundamentada en el cognitivismo, donde el enfoque de la enseñanza de la matemática centrado en la resolución de problemas se desarrolla mediante clases magistrales, en la cual, el estudiante participa en calidad de receptor de información "problematizada" por el docente. La enseñanza de la matemática se sucede dentro del ámbito del aula y limitado al uso del material instruccional proporcionado por el Ministerio de Educación. El proceso enseñanza-aprendizaje se sucede unidireccionalmente. En las EIB el aprendizaje activo de la matemática potencialmente puede producirse de forma natural y espontánea, siempre que se inscriba dentro del enfoque etnomatemático. Los estudiantes expresan empatía por el ejercicio del aprendizaje de la matemática basado en problemas propios de la comunidad y de su cotidianeidad. Para ello es fundamental que el docente domine el lenguaje nativo y planifique actividades que correspondan con el calendario comunal. Esa correspondencia, posibilita la satisfacción de los indicadores epistémicos, cognitivos, mediacionales y afectivos, de la idoneidad didáctica señalados por Godino et al. (2017) en tanto las actividades socioproductivas familiares y comunitarias en las que participa el estudiante cotidianamente, constituyen ámbitos de experiencia que demandan de su incorporación en la didáctica de la matemática enfocada en la solución de problemas.

En esta investigación el concepto matemático se flexibiliza dando cabida a elementos socioculturales de la etnia awajún durante el proceso enseñanza-aprendizaje de la matemática. Los estudiantes junto al docente exploran el entorno cultural y espacial para encontrar referentes que posibiliten el ejercicio matemático su interpretación y comprensión (Albanese y Perales, 2014).
En ese orden el objetivo de esta investigación fue establecer la relación entre el uso de aprendizaje activo y el desarrollo de competencias del área de matemática en los estudiantes del tercer grado de primaria de la Cultura Awajún, distrito de El Cenepa Amazonas Perú, 2018.

\section{METODOLOGÍA}

El diseño de investigación fue pre-experimental enfoque cuantitativo, aplicada y explicativa. Para la recolección de datos se aplicaron dos métodos: pre-experimental, de pretest/postest con un solo grupo y enseñanza observada. La población de estudio fueron estudiantes matriculados en el tercer grado de primaria de la I. E. N. ${ }^{\circ} 16348$-B de la localidad de Kusu Chico del Amazonas peruano con una muestra de 22 niños. Los instrumentos aplicados fueron la prueba pedagógica pretest/postest, es decir, una medición antes y después del experimento y la ficha de observación. Los resultados fueron procesados en el software SPSS. El método activo fue implementado en las sesiones de clase considerando dos elementos claves para el logro del objetivo propuesto:

- Acción social: Las actividades didácticas fueron diseñadas conforme a los signos y significados socialmente adquiridos y donde se desenvuelve cotidianamente el estudiante awajún. La enseñanza se realiza en lengua nativa y castellano como segunda lengua. El aprendizaje activo comprende una acción social donde el docente propone actividades colaborativas en las que el estudiante logre la construcción del conocimiento como consecuencia de la interacción social y desde su propia individualidad (Silva y Manturana, 
2017). En ese orden fueron desarrolladas actividades estructuradas en torno al calendario comunitario bajo el principio del tâjîmat pujut o del buen vivir.

- Matemática analógica: El proceso enseñanza aprendizaje se basa en una didáctica fundamentada en la dialógica que induce a que el estudiante sistematice el conocimiento adquirido desde situaciones reales del entorno, hasta que formalice de manera simbólica y logre el dominio de nociones de cantidad, cálculo, geometría y medición (Albánese y Perales, 2014). Para los estudiantes de origen awajún el entorno natural y las prácticas productivas tradicionales constituyen elementos donde aplican cotidianamente prácticas matemáticas. Basado en ello, se utilizan materiales estructurados y no estructurados para que el estudiante ejercite matemática analógica.

Transcurrido el primer mes del año escolar, fue aplicado un inventario de estilos de aprendizaje que sigue el modelo Felder y Silverman (1988), adaptado a niños de tercer grado, para determinar el estilo de aprendizaje VAK preferido por los estudiantes. El instrumento adaptado fue sometido a juicio de expertos. Para el establecimiento de los estilos de aprendizajes prevalentes en los sujetos del estudio, se aplicó el inventario de estilos de aprendizaje que responde a cinco interrogantes formuladas por Felder y Silverman (1988) en relación con las preferencias de los alumnos:

1. ¿Tipo de información preferida?

2. ¿Qué canal sensorial es más efectivo para percibir información?

3. ¿Cómo organiza la información?
4. ¿Cómo procesa la información?

5. ¿Cómo manifiesta el progreso hacia la comprensión?

El docente procedió a marcar las frecuencias en las respuestas observadas según la modalidad de aprendizaje prevalente: visual, auditiva, cenestésica.

Con base en ello fueron planificadas actividades para el desarrollo de las capacidades matemáticas esperadas en este nivel. Durante el transcurso del año escolar fueron desarrolladas actividades didácticas basadas en el aprendizaje activo e incorporación de principios etnomatemáticos, adaptados a los estilos de aprendizaje prevalentes en cada grupo. El sistema de numeración se trabajó en awajún y en castellano. Las pruebas pretest y postest fueron estructuradas en 20 preguntas discriminadas en 5 preguntas por contenido específico.

En ese orden los contenidos abordados comprenden:

1. Cantidad:

- Noción bilingüe del número natural:

Tabla 1. Numeración Awajún- Castellano

\begin{tabular}{ll}
\hline \multicolumn{1}{c}{ Awajún } & Castellano \\
\hline Makichik & uno \\
Jimag & dos \\
Kampatum & tres \\
Ipak usumat & cuatro \\
Makichik uwejan amua & cinco \\
Uwejan juinian makichik ijuk & seis \\
Uweja juinian jimajan ijuk & siete \\
Uweja juinian kampatum ijuk & ocho \\
Uwejan juinian ipak usumat ijuk & nueve \\
Uwejan mai amua & diez \\
Dawen makichik ijuk & once \\
Dawen jimajan ijuk & doce \\
Dawen kampatum ijuk & trece \\
Dawen ipak usumat ijuk & catorce
\end{tabular}




\begin{tabular}{ll}
\hline \multicolumn{1}{c}{ Awajún } & Castellano \\
\hline Dawen makichik amua & quince \\
Dawen juinian makichik ijuk & dieciséis \\
Dawen juinian jimajan ijuk & diecisiete \\
Dawen juinian kampatum ijuk & dieciocho \\
Dawen juinian ipak usumat ijuk & diecinueve \\
Dawe mai amua & veinte \\
Uweja kampatum amua & treinta \\
Uweja ipaksumat amua & cuarenta \\
Uweja makichik amua & cincuenta \\
Uweja makichik ijuk amua & sesenta \\
Uweja jimaja ijuk amua & setenta \\
Uweja mai amua & ochenta \\
Uweja ipaksumat ijuk amua & noventa \\
\hline
\end{tabular}

Fuente: Elaboración propia de la investigación.

- Valor posicional

- Orden en los números naturales

- Noción de fracción

- Fracciones equivalentes

Modelo de pregunta pretest del contenido cantidad:

a. El jueves salieron 6 canoas de pesca. Cada canoa lleva un motorista pescador y dos pescadores.

Pregunta: ¿Cuántos pescadores iban en cada canoa?

Respuesta: Awajún: Kampatum

Castellano: tres

Modelo de pregunta postest del contenido cantidad:

a. El jueves salieron de pesca dos canoas a motor, y cuatro canoas con remos.

Pregunta: ¿Cuántas canoas salieron a pescar?

Respuesta: Awajún: Uwejan juinian makichik ijuk

Castellano: seis
2. Cálculo:

- Adición y sustracción de números naturales

- Multiplicación de números naturales

- División de números naturales

- Adición, sustracción, multiplicación y división

- Múltiplos de un número natural

- Divisibilidad

Modelo de pregunta pretest del contenido cálculo:

b. El sábado Luis y su dúkug llevaron al mercado dos sacos de mama, para vender a 2 soles el kilo. Cada saco pesa 20 Kg. y lograron venderlo todo.

Pregunta ¿Cuánto lograron vender en soles?

Respuesta: Awajún: Uweja ipaksumat amua étsa

Castellano: cuarenta solesModelo de pregunta postest del contenido cálculo:

b. Las 2 canoas que salieron el lunes de pesca, trajeron 10 kilos de pescado cada una. Al llegar a la orilla vendieron a 4 soles cada kilo de pescado.

Pregunta: ¿Cuántos soles de pescado vendieron?

Respuesta: Awajún: Uweja mai amua étsa

Castellano: ochenta soles

3. Geometría:

- Orientación en el espacio

- Cuerpos geométricos

- Polígonos

- Círculo y circunferencia

Modelo de pregunta pretest del contenido geometría

c. En el mercado venden cestas octogonales de color blanco y marrón.

Pregunta: ¿Cuántos lados tienen las cestas? 
Respuesta: Awajún: Uweja juinian kampatum ijuk

Castellano: ocho

Modelo de pregunta postest del contenido geometría

c. La dúkug tapices con figuras hexagonales de paisajes.

Pregunta: ¿Cuántos lados tienen la figura?

Respuesta: Awajún: Uwejan juinian makichik ijuk

Castellano: seis

4. Medidas:

- Longitud

- Capacidad

- Peso

- Sistema monetario

- Tiempo: El día en awajún se estructura en doce períodos de tiempo:

Tabla 2. Medida de Tiempo awajún-castellano

\begin{tabular}{|c|c|c|}
\hline Awajún & Castellano & Hora \\
\hline Étsa jiínne & Salida del sol & 6 a. m. \\
\hline Étsa takaúnne & Sol alzado & 8 a. m. \\
\hline $\begin{array}{l}\text { Étsa tajiágtatak } \\
\text { wajasú }\end{array}$ & Sol casi divide el día & 10 a. m. \\
\hline Étsa tajímai & Mitad el día & $12 \mathrm{pm}$ \\
\hline Étsa tegáwai & $\begin{array}{l}\text { El sol comienza a } \\
\text { declinar }\end{array}$ & 2 p. m. \\
\hline $\begin{array}{l}\text { Agkuántai cuando } \\
\text { es tarde }\end{array}$ & Tarde & 4 p. m. \\
\hline Étsa akáewai & Puesta del sol & $6 \mathrm{p.m}$. \\
\hline Kiáje & Oscuridad & 8 p. m. \\
\hline Áehaet & Mitad de la noche & 12 p. m. \\
\hline $\begin{array}{l}\text { Tsawán íjag el día } \\
\text { visita }\end{array}$ & Proximidad del día & 2 a. m. \\
\hline Tsawáwai & Amanecer & 4 a. m. \\
\hline
\end{tabular}

Fuente: Elaboración propia de la investigación.

Modelo de pregunta pretest del contenido medidas:

d. Laya, Katán y Mashián estudian sexto grado. Laya siempre se encuentra de primera en la fila, Katán de tercero y Mashián de último.

Pregunta: Si las filas se organizan en orden de tamaño de menor a mayor, ¿quién de los tres es el más alto y quien es el más bajo?

Respuesta: Awajún: Mashián niyai esajam, Laya niyai sutag

Castellano: Mashián es el más alto y Laya la más baja.

Modelo de pregunta postest del contenido medidas:

d. Liliana, Akáchu y Anchuíg son hermanos. Liliana nació en el año 2008, Akáchu nació en el año 2011 y Anchuíg es un año mayor que Liliana.

Pregunta: ¿Quién de los hermanos es mayor?

Respuesta: Akáchu.

Posterior a la prueba pretest, las sesiones de clase se desarrollaron a través del método activo, problematizando la realidad del entorno awajún en la comunidad de Kusu Chico. Las sesiones inician problematizando una realidad concreta por el docente, en lengua awajún y castellano. Cada sesión fue registrada en fichas de observación. Estos ejercicios comprendieron actividades de campo, como el cálculo de la producción de mama (yuca) tanto por unidades, como por peso. Así como, contar y calcular mediante operaciones aditivas, sustractivas, multiplicativas y de división, los resultados comunitarios y familiares de las diversas actividades económicas en las que participan. Los estudiantes junto al docente visitaron unidades de producción agrícola, artesanal y pesquera, para identificar actores y distinguir el tipo de actividad económica en la que participa su familia. Posteriormente, se les asigna tareas 
de levantamiento de información mediante consulta a sus padres y familiares, al responder preguntas como:

¿Cuántos pescadores iban en la canoa que salió hoy de pesca?, ¿Cuántos pescados trajeron?, ¿Cuántos pescados le tocó a cada uno?, ¿Cuántas matas de mama están sembradas en el conuco familiar?, ¿Cuántos kilos pesó la mama más grande que sacaron la última vez?, ¿En cuánto se debe dividir el peso de la mama, para que le toque un trozo igual a cada miembro de la familia? ¿Qué formas tienen las tinajas que hacen en Kuju Chico?, ¿Qué tinaja le gusta más a tu Dúkug (mamá) la ovalada o la de cuello cilíndrico?

\section{ANÁLISIS Y RESULTADOS}

El objetivo de esta investigación fue establecer la relación entre el uso de aprendizaje activo y el desarrollo de competencias en el área de matemática en los niños de la cultura Awajún, estudiantes de tercer grado de primaria, provincia Condorcanqui, distrito de El Cenepa, departamento Amazonas, Perú. Durante las primeras 4 semanas del curso se logró identificar los estilos de aprendizaje preferido por los alumnos: $57 \%$ visuales, $29 \%$ cenestésicos y $12 \%$ auditivos. Ello no excluye que los estudiantes recurran a estilos multimodales, solo indica cuál de los estilos de aprendizaje prefieren por sobre los otros.

En el transcurso del primer trimestre del año escolar se desarrollaron actividades orientadas hacia la promoción de comunicación eficiente estudiante-docente, mediante estrategias didácticas que fomentaran la resolución de conflictos semióticos (Godino, 2011). Esta fase persiguió el objetivo de establecer comunidad semántica entre estudiantes y docentes, aunado a la identificación de los estilos de aprendizaje que prevalecen entre los estudiantes de la muestra. La prevalencia de estudiantes quienes prefieren estilos visuales y cenestésicos de aprendizaje indujo hacia la planificación de actividades de campo por el docente que derivaron en la problematización de la realidad y la propuesta de estrategias matemáticas apara su solución por los estudiantes.

Las Tablas 3 al 6, en la tercera y cuarta columnas, expresan resultados de la prueba pretest por capacidad. La prueba indica que no hubo estudiantes del $3^{\circ}$ grado de primaria de la Institución IEB N. ${ }^{\circ} 16348-B$ de la localidad de Kusu Chico del distrito de El Cenepa, Amazonas, quienes alcanzaran los niveles de logro ni logro destacado en las capacidades matemáticas exigidas por el currículo nacional: matematiza situaciones, comunica y representa ideas matemáticas, elabora y usa estrategias, y, razona y argumenta generando ideas matemáticas. Al retornar a las sesiones de aula, se observó la aplicación de estrategias didácticas correspondientes al aprendizaje activo desde el enfoque de solución de problemas, tal como lo encontraron los investigadores de la Universidad ICESI (2017). Los estudiantes recurrieron a elementos del entorno para realizar ejercicios de matematización, expresión y representación de situaciones del entorno aplicando etnomatemática individual y colectivamente. Todo ello fundamentado en la experiencia de campo y en su vida cotidiana, lo que derivó en el desplazamiento desde el nivel de inicio y proceso hasta los niveles de logro y logro destacado por el $72 \%$ de los estudiantes en el postest (Ver Tablas 3 y 4 ). 
Tabla 3. Matematiza situaciones

\begin{tabular}{lccccc}
\hline \multicolumn{1}{c}{ Nivel } & Rango & \multicolumn{2}{c}{ Prueba pretest } & \multicolumn{2}{c}{ Prueba postest } \\
& & Frecuencia & \% & Frecuencia & \% \\
\hline Inicio & $(1-10)$ & 10 & 45.45 & 3 & 14 \\
Proceso & $(11-12)$ & 12 & 54.54 & 3 & 14 \\
Logro previsto & $(13-16)$ & 0 & 0 & 5 & 22 \\
Logro destacado & $(17-20)$ & 0 & 0 & 11 & 50 \\
Total & & 22 & 100 & 22 & 100 \\
\hline
\end{tabular}

Fuente: Elaboración propia de la investigación.

Tabla 4. Comunica y representa ideas matemáticas

\begin{tabular}{lccccc}
\hline \multicolumn{1}{c}{ Nivel } & Rango & \multicolumn{2}{c}{ Prueba pretest } & \multicolumn{2}{c}{ Prueba postest } \\
& & Frecuencia & Porcentaje & Frecuencia & Porcentaje \\
\hline Inicio & $(1-10)$ & 9 & 40.59 & 3 & 14 \\
Proceso & $(11-12)$ & 13 & 59.09 & 3 & 14 \\
Logro previsto & $(13-16)$ & 0 & 0 & 5 & 22 \\
Logro destacado & $(17-20)$ & 0 & 0 & 11 & 50 \\
Total & & 22 & 100 & 22 & 100 \\
\hline
\end{tabular}

Fuente: Elaboración propia de la investigación.

Los resultados anteriores, concuerdan con las investigaciones de Labrador y Andreu (2015), quienes destacaron el desempeño docente como promotor del aprendizaje activo, lo que deriva en que los estudiantes asuman roles de prosumidores de conocimiento. En ese rol los estudiantes se inician en los procesos de análisis, reflexión y discusión interpares de problemas cotidianos al aplicar principios matemáticos e interpretar resultados. Para los niños awajún se expresa en matematizar situaciones y proponer estrategias de solución a problemas de su entorno individual y colectivo, lo que se observa en los resultados postest. (Ver Tablas 5 y 6).

Tabla 5. Elabora y usa estrategias

\begin{tabular}{lccccc}
\hline Nivel & Rango & \multicolumn{2}{c}{ Prueba pretest } & \multicolumn{2}{c}{ Prueba postest } \\
& & Frecuencia & $\%$ & Frecuencia & $\%$ \\
\hline Inicio & $(1-10)$ & 9 & 40.59 & 3 & 14 \\
Proceso & $(11-12)$ & 13 & 59.09 & 3 & 14 \\
Logro previsto & $(13-16)$ & 0 & 0 & 5 & 22 \\
Logro destacado & $(17-20)$ & 0 & 0 & 11 & 50 \\
Total & & 22 & 100 & 22 & 100 \\
\hline
\end{tabular}

Fuente: Elaboración propia de la investigación. 
Tabla 6. Razona y argumenta generando ideas matemáticas

\begin{tabular}{lccccc}
\hline \multirow{2}{*}{ Nivel } & Rango & \multicolumn{2}{c}{ Prueba pretest } & \multicolumn{2}{c}{ Prueba postest } \\
& & Frecuencia & $\%$ & Frecuencia & $\%$ \\
\hline Inicio & $(1-10)$ & 10 & 45.45 & 3 & 14 \\
Proceso & $(11-12)$ & 12 & 54.54 & 3 & 14 \\
Logro previsto & $(13-16)$ & 0 & 0 & 5 & 22 \\
Logro destacado & $(17-20)$ & 0 & 0 & 11 & 50 \\
Total & & 22 & 100 & 22 & 100 \\
\hline
\end{tabular}

Fuente: Elaboración propia de la investigación.

Los estudiantes awajún del tercer grado de la IE N. ${ }^{\circ} 16348$-B de la localidad de Kusu Chico demostraron empatía por la práctica matemática en la resolución de problemas cotidianos asociados a su familia y comunidad, en tanto aplicaron los conocimientos sobre problemáticas que les eran conocidos. La contrastación entre lo observado en las prácticas de campo con la información obtenida en el entorno familiar, referida a las actividades productivas locales, estimuló al $72 \%$ de los estudiantes en el desarrollo de la capacidad de comunicar y representar ideas matemáticas, lo que se reflejó en los resultados postest. La investigación de Zumaeta, Fuster y Ocaña (2018) coincide con los resultados de este estudio, por cuanto desde la perspectiva afectiva en la enseñanza de la matemática, señalan que, la atención personalizada del estudiante en función de sus estilos de aprendizaje y el reconocimiento de las cualidades distintivas de cada uno por el docente, despierta el interés hacia la asignatura y el desempeño de roles activos por el estudiantado.

Obsérvese que la didáctica fundamentada en aprendizaje activo dentro de los estudiantes awajún de tercer grado de primaria en Kusu Chico, comprendió la incorporación de materiales estructurados y no estructurados disponibles en el aula y en el entorno. La idoneidad mediacional de los materiales proporcionados por el entorno instrumentó al estudiante con referentes para la problematización de realidades cotidianas. La idoneidad epistémica y cognitiva (Godino, 2017), de las estrategias didácticas fundamentadas en el aprendizaje activo, se observan en el desplazamiento de los estudiantes desde los niveles de inicio y proceso en las capacidades matemáticas descritas en la Tabla 7. El $72 \%$ de los estudiantes alcanzaron los niveles de logro y logro destacado en todas las capacidades matemáticas contenidas en el currículo de EIB (Minedu, 2016c).

Tabla 7. Competencias matemáticas generales

\begin{tabular}{llllll}
\hline \multicolumn{1}{c}{ Nivel } & Rango & \multicolumn{2}{c}{ Prueba de entrada } & \multicolumn{2}{c}{ Prueba de salida } \\
& & Frecuencia & \% & Frecuencia & \% \\
\hline Inicio & $(1-10)$ & 9 & 40.59 & 3 & 14 \\
Proceso & $(11-12)$ & 13 & 59.09 & 3 & 14 \\
Logro previsto & $(13-16)$ & 0 & 0 & 5 & 22 \\
Logro destacado & $(17-20)$ & 0 & 0 & 11 & 50 \\
\hline Total & & 22 & 100 & 22 & 100 \\
\hline
\end{tabular}

Fuente: Elaboración propia de la investigación. 
Para los estudiantes del cuarto grado de la cultura awajún en Kusu Chico, el uso analógico de la matemática constituye un proceso aprendido en los espacios de socialización primaria, en lo concerniente a las capacidades de contar, calcular aditiva y sustractivamente, $y$, medir. Utiliza como referente a la producción conuquera, artesana y pesquera, el $72 \%$ de los estudiantes demostraron la capacidad de calcular lo producido en actividades económicas como la pesca y la siembra de mama, así como distribuir los productos, mediante operaciones divisivas. En lo referido a la abstracción de estos procesos llevado a situaciones imaginarias en contextos distintos al propio, se observó que la capacidad de comunicarse y representar ideas matemáticas se dificulta, por cuanto tienden a recurrir hacia los referentes propios reiteradamente. Los estudiantes quienes alcanzaron el logro destacado se distinguen por los niveles de abstracción y la capacidad de argumentación matemática, en contexto diversos.

En lo referente a las capacidades geométricas el aprendizaje activo como didáctica se demostró eficiente, por cuanto al contar con el 57 \% (13) estudiantes quienes prefieren el aprendizaje visual y un $29 \%$ (6) estudiantes de preferencia cenestésica, el reconocimiento e identificación apropiada de formas geométricas en el entorno se produjo espontáneamente en el $89 \%$ (19) de los casos. El $12 \%$ de los alumnos, quienes prefieren el estilo auditivo de aprendizaje fueron receptivos y la totalidad de ellos alcanzaron el nivel de logro o logro destacado. El aprendizaje activo que utiliza materiales no estructurados demostró idoneidad mediacional, afectiva y ecológica en la enseñanza de la matemática para estudiantes de tercer grado de educación primaria.

\section{Prueba de hipótesis}

La prueba de hipótesis evidenció que el p-valor (000) es menor al nivel de significancia de 0.05 en consecuencia se rechaza la hipótesis nula (Ho) y se acepta la hipótesis alterna (Ha). Se concluye que, el uso del aprendizaje activo influye favorablemente en el logro de competencias en el área de matemática en los niños de tercer grado de educación primaria de la Institución Educativa N. ${ }^{\circ}$ 16348-B Kusu Chico, distrito de El Cenepa, Amazonas 2018.

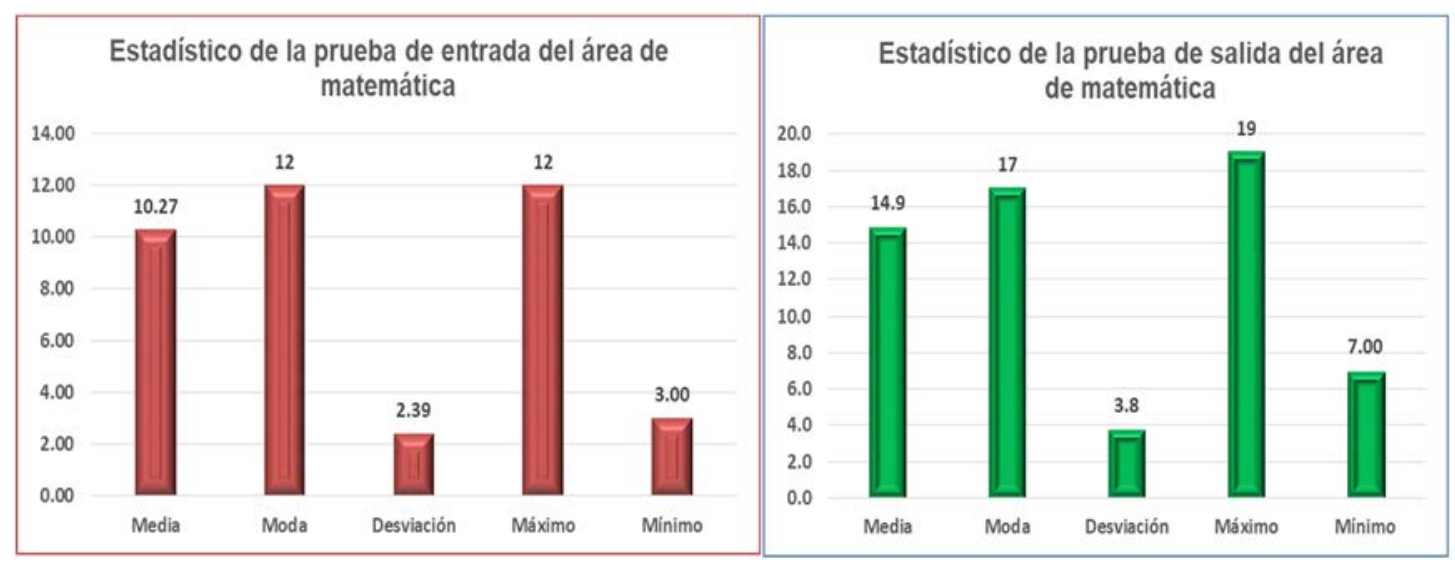

Gráfico 1. Estadístico del área de Matemática Niños de $3^{\circ}$ Grado Fuente: Elaboración propia de la investigación. 


\section{CONCLUSIONES}

El uso del aprendizaje activo influye significativamente en el logro de las capacidades y el alcance de las competencias matemáticas por los estudiantes del tercer grado de primaria de la Institución Educativa N. ${ }^{\circ}$ 16348-B Kusu Chico, distrito de El Cenepa, Amazonas 2018; con un nivel de significancia de 0.05 al $95 \%$.

La enseñanza observada evidenció la necesidad de resolver de forma temprana los conflictos semióticos, como paso previo para la intercomprensión estudiante-docente. $\mathrm{La}$ intercomprensión es indispensable para que los estudiantes se incentiven hacia el ejercicio de tareas de prosumidores de conocimiento, fundamentado en la problematización de su realidad y la del entorno, que en esencia comprende el ejercicio de aprendizaje activo. Para los niños awajún, el desempeño como prosumidores se expresa en matematizar situaciones, dentro de los espacios socioproductivos donde su familia y comunidad hacen vida. Aunado a ello, debaten sobre soluciones a los problemas fundamentándose en su experiencia de vida, que avanza paulatinamente hacia la proposición de soluciones alternas y el ejercicio de la abstracción. La didáctica del aprendizaje activo de la matemática en niños de la comunidad awajún evidenció la presencia de indicadores de idoneidad didáctica, que fueron confirmados con los resultados de la prueba postest. Es fundamental continuar realizando estudios sobre la enseñanza de la matemática en entornos de las escuelas interculturales bilingües amazónicas, que, dadas las especificidades de cada etnia, lo intrincado de los lugares donde se ubican y el reducido número de docentes-investigadores en los espacios interculturales, aún denota la existencia de brechas que demandan satisfacción.

\section{RECONOCIMIENTOS}

Trabajo elaborado en bajo los auspicios de la Facultad de Educación Intercultural y Humanidades, Universidad Nacional Intercultural de la Amazonia. Línea de Investigación Innovación Pedagógica.

\section{DECLARACIÓN DE LA CONTRIBUCIÓN DE LOS AUTORES}

El porcentaje total de contribución para la conceptualización, preparación y corrección de este artículo fue el siguiente: C.E.A. 45 \%, M.P.K.M. 30 \% y J.R.C.L. 25\%.

\section{DECLARACIÓN DE DISPO- NIBILIDAD DE LOS DATOS}

Los datos que respaldan los resultados de este estudio serán puestos a disposición por el autor correspondiente C.E.A., previa solicitud razonable.

\section{REFERENCIAS}

Albánese, V.; \& Perales, F. (2014). Pensar matemáticamente: una visión etnomatemática de la práctica artesanal soguera. Revista latinoamericana de investigación en matemática educativa, 17(3), 261-288. https://doi. org/10.12802/relime.13.1731

Borracci, R.; Manente, D.; Tamini, S.; Dvorkin, M.; Arribalzaga, E.; Grancelli, H.; \& Galli, A. (2015). Learning styles preferences from elementary school to medical postgraduate. FEM: Revista de la Fundación Educación Médica, 18(2), 123-129. Recuperado de https://dx.doi. org/10.4321/S2014-98322015000200008

Burneo, R. (2018). Territorio Integral Indígena, una propuesta awajún. Iztapalapa. Revista de ciencias sociales y humanidades, 39(85), 33-57. Recuperado de https://dx.doi.org/10.28928/ revistaiztapalapa/852018/atc2/burneomendozar 
Felder, R., \& Silverman, L.K. (1988). Learning and teaching styles in engineering education. Journal of Engineering Education, 78(7), 674-681. Recuperado de https:// www.engr.ncsu.edu/wp-content/uploads/ drive/1QP6kBI1iQmpQbTXL-08HS10PwJ5BYnZW/1988-LS-plus-note.pdf

Godino, J. (2011). Indicadores de idoneidad didáctica de procesos de enseñanza y aprendizaje de las matemáticas1. XIII Conferência Interamericana de Educação Matemática (CIAEM-IACME), Recife, Brasil Recuperado de: https://www.ugr.es/ jgodino/eos/jdgodino indicadores_idoneidad.pdf

Godino, J. (2017). Construyendo un sistema modular e inclusivo de herramientas teóricas para la educación matemática. En J. M. Contreras, P. Arteaga, G. R. Cañadas, M.M. Gea, B. Giacomone y M. M. López-Martín (Eds.). Actas del Segundo Congreso International Virtual sobre el Enfoque Ontosemiótico del Conocimiento y la Instrucción Matemáticos. (pp.2-20). Granada, España. Recuperado de https://www.semanticscholar. org/paper/Construyendo-un-sistema-modular-e-inclusivodeparaGodino/0647bee2f3e682e$712 \mathrm{e} 19 \mathrm{bacd} 81 \mathrm{db} 755 \mathrm{~b} 9 \mathrm{e} 61 \mathrm{e} 3 \mathrm{c}$

Godino, J.; Giacomone, B.; Batanero, C.; \& Font, V. (2017). Enfoque Ontosemiótico de los Conocimientos y Competencias del Profesor de Matemáticas. Boletim de Educação Matemática, 31(57), 90-113. https://doi. org/10.1590/1980-4415v31n57a05

Instituto Nacional de Estadística e Informática [INEI]. (2018). Resultados Definitivos de III Censo de Comunidades Nativas 2017. Lima: INEI Recuperado de: https://www.inei.gob.pe/media/MenuRecursivo/publicaciones_digitales/ Est/Lib1598/

Laboratorio Latinoamericano de Evaluación de la Calidad de la Educación (LLECE). (2016). Informe de Resultados del Tercer Estudio Regional Comparativo Explicativo (TERCE). REICE. Revista Iberoamericana Sobre Calidad, Eficacia y Cambio en Educación, 14 (4), 9-32. https://doi.org/10.15366/ reice2016.14.4.001

Labrador, M.; \& Andreu, M. (2008). Metodologías activas. Valencia, ES: Ediciones Universidad Politécnica de Valencia. Recuperado de: http://www.upv.es/diaal/publicaciones/Andreu-Labrador12008_Libro\%20Metodologias_Activas.pdf
Ministerio de Educación del Perú [Minedu]. (2015a). Resultados de la Evaluación Censal de Estudiantes 2015. Recuperado de: http://umc. minedu.gob.pe/wp-content/uploads/2016/03/ Resultados-ECE-2015.pdf.

Ministerio de Educación del Perú [Minedu]. (2015b). Matemáticas en Educación Intercultural bilingüe. Lima: Minedu. Recuperado de: http://repositorio.minedu.gob.pe/ handle/123456789/4864

Ministerio de Educación del Perú [Minedu]. (2016a). Resultados de la Evaluación Censal de Estudiantes 2016. Lima: Minedu. Recuperado de: http://umc.minedu.gob.pe/equidad-y-oportunidades-de-aprendizaje-en-2-grado-de-secundaria-evidencias-de-la-ece-2016/

Ministerio de Educación del Perú [Minedu]. (2016b). ¿Qué logran nuestros estudiantes en ECE? Informe para la Institución Educativa. Lima: Minedu. Recuperado de http://umc. minedu.gob.pe/wp-content/uploads/2017/04/ Informe-para-la-Instituci\%C3\%B3n-Educativa-ECE-2016.pdf

Ministerio de Educación del Perú [Minedu]. (2016c). Programa curricular de educación primaria. Lima: Minedu. Recuperado de: http://www. minedu.gob.pe/curriculo/pdf/programa-nivel-primaria-ebr.pdf.

Ministerio de Educación del Perú [Minedu]. (2018). Resultados ECE y Evaluación Muestral. Lima: Minedu Recuperado de: http://umc.minedu.gob.pe/resultados-ece-2018/

Ministerio de la Cultura del Perú (2018). Base de Datos de Pueblos Indígenas u originarios. Ministerio de Cultura. Recuperado de: https://bdpi.cultura.gob.pe/localidades/ kusu-chico-o-cusu-chico

Mota, A.; Henríquez, A.; \& Oliveira, H. (2016). El Desarrollo de la Capacidad de Resiliencia Matemática: La Voz de los Estudiantes Sobre el Uso de Las TIC en la Aula. Electronic Journal of Research in Educational Psychology, 14(1), 67-88. Recuperado de: DOI:10.25115/ ejrep.38.15041.

OCDE. (2014). Resultados Pisa 2012 en FOCO. Recuperado de: https://www.oecd.org/pisa/ keyfindings/PISA2012_Overview_ESP-FINAL.pdf

OCDE. (2016). Pisa 2015 Resultados Clave. Recuperado de: https://www.oecd.org/pisa/pisa2015-results-in-focus-ESP.pdf 
OCDE. (2006). Pisa 2006 Marco de la Evaluación. Conocimientos y habilidades en ciencias, matemáticas y lectura. Recuperado de http:// www.oecd.org/pisa/39732471.pdf

Osores, F.; Rojas, J.; \& Lara, C. (2012). Minería informal e ilegal y contaminación con mercurio en Madre de Dios: Un problema de salud pública. Acta Médica Peruana, 29(1), 38-42. Recuperado de: http://www.scielo. org.pe/scielo.php?script=sci_arttext\&pi$\mathrm{d}=\mathrm{S} 1728-59172012000100012$

Salas, S. (2018). Articulación de las matemáticas mapuche y escolar en el caso de los conocimientos aritméticos. Tesis Doctoral. Universidad de Granada, Granada, España. Recuperado de: https://digibug.ugr.es/ handle/10481/54976

Silva, J.; \& Maturana, D. (2017). Una propuesta de modelo para introducir metodologías activas en educación superior. Innov. educ. 7(73), 117-131. Recuperado de: http://www.scielo. org.mx/scielo.php?script=sci_arttext\&pi$\mathrm{d}=\mathrm{S} 1665-26732017000100117$

Solar, H.; \& Deulofeu, J. (2016). Condiciones para promover el desarrollo de la competencia de argumentación en el aula de matemáticas. $B o$ lema, Rio Claro (SP), 30(56), 1092-1112. https://doi.org/10.1590/1980-4415v30n56a13
UNESCO \& Laboratorio Latinoamericano de Evaluación de la Calidad de la Educación (LLECE) (2008). SERCE: Los aprendizajes de los estudiantes de América Latina y el Caribe. Primer reporte de los resultados del Segundo Estudio Regional Comparativo y Explicativo. Santiago: OREALC/UNESCO. Recuperado en: http://unesdoc.unesco.org/ images/0016/001606/160660S.pdf

UNESCO \& Laboratorio Latinoamericano de Evaluación de la Calidad de la Educación (LLECE) (2015). TERCE: Los aprendizajes de los estudiantes de América Latina y el Caribe. Tercer reporte de los resultados del Segundo Estudio Regional Comparativo y Explicativo. Santiago: OREALC/UNESCO. Recuperado en: https://unesdoc.unesco.org/ark:/48223/ pf0000243983

Universidad ICESI. (2017). PEI Aprendizaje Activo. Cali: Editorial Universidad Icesi. Recuperado de: https://www.icesi.edu.co/contenido/pdfs/ pei/PEI-Aprendizaje-Activo.pdf

Zumaeta, S.; Fuster, D.; \& Ocaña Y. (2018). El afecto pedagógico en la didáctica de la matemática - Región Amazonas desde la mirada fenomenológica. Propósitos y Representaciones, 6(1), 409-462. https://doi.org/10.20511/ pyr2018.v6n1.200

\section{(c) (i) $(9)$}

Método activo en el desarrollo de competencias matemáticas en niños de la cultura Awajún, Perú (Abdías Chávez-Epiquén • Ketty Marilú Moscoso-Paucarchuc • Juan Raúl Cadillo-León). Uniciencia is protected by Attribution-NonCommercial-NoDerivs 3.0 Unported (CC BY-NC-ND 3.0) 